\title{
THE GROUP OF AUTOMORPHISMS OF A CLASS OF FINITE $p$-GROUPS
}

\author{
BY
}

ARYE JUHÁSZ

\begin{abstract}
ABSTRACr. Let $G$ be a finite $p$-group and denote by $K_{i}(G)$ the members of the lower central series of $G$. We call $G$ of type $(m, n)$ if (a) $G$ has nilpotency class $m-1$, (b) $G / K_{2}(G) \cong \mathbf{Z}_{p^{n}} \times \mathbf{Z}_{p^{n}}$ and $K_{i}(G) / K_{i+1}(G) \cong \mathbf{Z}_{p^{n}}$ for every $i, 2 \leqslant i \leqslant n-1$. In this work we describe the structure of $\operatorname{Aut}(G)$ and certain relations between $\operatorname{Out}(G)$ and $G$.
\end{abstract}

Introduction. N. Blackburn considered in [1] a special class of finite $p$-groups, the $p$-groups of maximal class. Our aim here is to determine the structure of the automorphism group of a wider class of finite $p$-groups, groups $G$ with nilpotency class $m-1$, such that $G / K_{2}(G) \cong \mathbf{Z}_{p^{n}} \times \mathbf{Z}_{p^{n}}$ and, for $2 \leqslant i \leqslant m-1$, $K_{i}(G) / K_{i+1}(G) \cong \mathbf{Z}_{p^{n}}$. We call such groups $G$ of type $(m, n)$. Here $K_{i}(G)$ denotes the $i$ th member of the descending central series of $G$ and $m, n$ are positive natural numbers, $m>2$. (Thus a $p$-group of maximal class of order $p^{m}$ is of type $(m, 1)$ ). Such groups were dealt with in [2] and independently in [5]. It becomes clear right at the beginning of our investigation that if $G$ is a $p$-group of type $(m, n)$ then $\operatorname{Aut}(G)$ has a normal Sylow $p$-subgroup $P$ and $\operatorname{Aut}(G) / P$ is isomorphic to a subgroup of $\mathbf{Z}_{p-1} \times \mathbf{Z}_{p-1}$ (Theorem 1.12). So, naturally, we focus on the structure of $P$ and prove that, roughly, in the splitting of $P$ to three parts by $\bar{G} \triangle B \triangle P$, the size of $B / \bar{G}$ is bounded from below by a number which depends on $Z\left(G_{1}\right)$ and $G_{1}^{\prime}$ (Theorem 2.3). Under certain conditions this means that $G$ has many outer automorphisms. Here $\bar{G}$ denotes the group of the inner automorphisms of $G, B$ stands for the subgroup of $\operatorname{Aut}(G)$ of all automorphisms which fix $G / K_{2}(G)$ elementwise and $P / B$ is a subgroup of $\operatorname{GL}\left(2, p^{n}\right)$ which is isomorphic to $\operatorname{Aut}\left(G / K_{2}(G)\right)$.

In $\S 3$ we deal with metabelian $p$-groups of type $(m, n)$. For these groups our results are more precise: We determine the upper and lower central series of $P$ under certain conditions (which are satisfied by metabelian $p$-groups of maximal class) and show that $B / \bar{G}$ has a very similar structure to that of a subgroup of $K_{2}(G)$. We also give a lower bound for $B / \bar{G}$ in terms of $m, n$ and $p$ (Theorem 3.2). Here we are working in the endomorphism ring of $K_{2}(G)$ generated by $G / K_{2}(G)$ and we use an idea of M. Lazard [8] exploited in [6].

We close by $\S 4$ with sharpening our results obtained in $\$ \S 2$ and 3 for $p$-groups of maximal class.

Received by the editors December 19, 1978 and, in revised form, February 18, 1981.

1980 Mathematics Subject Classification. Primary 20D45; Secondary 20 D 15.

Key words and phrases. Finite $p$-group, automorphism group, metabelian group, power-structure of $p$-groups, commutator-structure of $p$-groups, $p$-groups of maximal class. 
ACKNOWLEDGements. This work is a part of my doctoral thesis done under the kind supervision of Professor A. Mann. I wish to express my thanks to Professor Mann for his aid during the preparation of it. I am indebted to the referee for pointing out an error in the original version.

0. Notation. We follow the notation of $[4$, III]. Let $G$ be a finite group. For every $a, b \in G$ define $[a, 0 b]=a$ and for every $0<n \in \mathbf{Z}$ define

$$
[a, n b]=[[a,(n-1) b], b] \text {. }
$$

Here $[c, b]=c^{-1} b^{-1} c b$ for every $c, b \in G$. For subsets $X$ and $Y$ of $G$ let $\langle X, Y\rangle$ be the subgroup of $G$ generated by $X$ and $Y$ in $G$ and $[x, y]=\langle[x, y] \mid x \in X, y \in Y\rangle$. For every $i>1$ let $K_{i}(G)$ and $Z_{i}(G)$ be the $i$ th member of the descending and ascending central series of $G$, respectively. Abbreviate $Z_{1}(G)$ by $Z(G)$ and the nilpotency class of $G$ by $\operatorname{cl}(G)$. Denote by $F(G)$ and $\Phi(G)$ Fitting and the Frattini subgroup of $G$, respectively (see [4, III]). Let $p$ be a fixed prime number. For every natural $n, \Omega_{n}(G)=\left\langle x \in G \mid x^{p^{n}}=1\right\rangle, \mho_{n}(G)=\left\langle x^{p^{n}} \mid x \in G\right\rangle$ and abbreviate the exponent of $G$ by $\exp (G)$. Aut $(G)$ stands for the group of automorphisms of $G$ and if $G$ is abelian then $\operatorname{End}(G)$ stands for the endomorphism ring of $G$. For every $\sigma \in \operatorname{Aut}(G)$ and $x \in G$ we denote the action of $\sigma$ on $x$ by $x^{\sigma}$ and write $[x, \sigma]$ for $x^{-1} x^{\sigma}$. These commutators are defined in the semidirect product of $G$ by $\operatorname{Aut}(G)$; hence all the rules for commutators hold for them. Write " $H \triangle G$ " for " $H$ is a normal subgroup of $G$ ".

For every element (subgroup) $x(X)$ of $G$ denote by $\bar{x}(\bar{X})$ the inner automorphism (group) of $G$ induced by $x(X)$. We shall use freely the following identities of commutators [4, III, pp. 253, 254]: For every $a, b, c \in G$ :

$(\alpha)\left[a, b^{-1}\right]=[a, b]^{-b^{-1}}$,

$(\beta)[a, b c]=[a, c][a, b]^{c}$,

$(\gamma)[a b, c]=[a, c]^{b}[b, c]$,

( $\delta)\left[a, b^{-1}, c\right]^{b}\left[b, c^{-1}, a\right]^{c}\left[c, a^{-1} b\right]^{a}=1$ (Witt's identity).

Finally, we recall the collection formula [4, III, p. 317]: For every $a, b \in G$,

$$
(a b)^{p^{n}}=a^{p^{n}} b^{p^{n}} c_{2}^{\left(p^{n}\right)} \ldots c_{t}^{\left(p^{n}\right)} \ldots c_{p^{n}}, \quad c_{t} \in K_{t}(\langle a, b\rangle)
$$

1. Basic results. Let $G$ be a $p$-group of type $(m, n), m \geqslant 4$. For $i \geqslant 2$ define $G_{i}=K_{i}(G)$ and for $i=1$ define $G_{1}$ by $G_{1} / G_{4}=C_{G / G_{4}}\left(G_{2} / G_{4}\right)$. If there exists a natural number $k$ such that, for every $i, j \geqslant 1,\left[G_{i}, G_{j}\right] \leqslant G_{i+j+k}$, then following $\mathrm{N}$. Blackburn [1], we say that $G$ has degree of commutativity $k$.

We shall need the following basic properties of $p$-groups of type $(m, n)$, which we state without proof. They follow easily from the results of N. Blackburn in [1].

Let $G$ be a $p$-group of type $(m, n), m \geqslant 4$. Then

(1.1) There exists an element $s_{1} \in G$ such that $G_{1}=G_{2}\left\langle s_{1}\right\rangle$ and $G=\left\langle s, s_{1}\right\rangle$, for every $s \in G \backslash G_{1} \Phi(G)$. If for $i \geqslant 2$ we define $s_{i}=\left[s_{i-1}, s\right]$ then $G_{i}=\left\langle G_{i+1}, s\right\rangle$. Every element in $G$ can be expressed uniquely by $s^{\alpha_{0}} s_{1}^{\alpha_{1}} \ldots s_{t}^{\alpha_{t}} \ldots s_{m-1}^{\alpha_{m-1}}, \alpha_{t} \in \mathbf{Z}$, $0 \leqslant \alpha_{t}<p^{n}$. 
(1.2) For every $x \in G \backslash G_{1} \Phi(G), x^{p^{n}} \in G_{m-1}$ and $C_{G}(x)=\langle x\rangle Z(G)$.

(1.3) For every $x \in G \backslash G_{1} \Phi(G),[x, G]=G_{2}$.

(1.4) $Z_{i}(G)=G_{m-i}$, for $1<i<m-1$.

(1.5) If $m \leqslant p+1$, then $\exp \left(G_{2}\right)=\exp \left(G / G_{m-1}\right)=p^{n}$.

(1.6) If $m \geqslant p+2$, then $\mho_{1}\left(G_{i}\right) \leqslant G_{i+p-1}$ and, for $n=1, \mho_{1}\left(G_{i}\right)=G_{i+p-1}$.

(1.7) If $m \geqslant p+2$, then

$$
s_{1}^{p^{n}} \equiv s_{p}^{\left(p_{p}^{n}\right)} \bmod \left(G_{p+1}\right)
$$

(1.8) If $G$ is metabelian then $G$ has degree of commutativity $\geqslant 1$.

(1.9) Let $G$ be metabelian and let $s \in G \backslash G_{1} \Phi(G)$ and for $i \geqslant 1$ let $s_{i}$ be as defined in (1.1). Then

(a) If $\left[s_{1}, s_{2}\right]=s_{m-k}^{x_{k}} \ldots s_{m-1}^{x_{1}}$ then $\left[s_{1}, s_{i}\right]=s_{m-k+i-2}^{x_{k}} \ldots s_{m-1}^{x_{i-1}}$, for every $i \geqslant 2$.

(b) The following are defining relations for $G_{2}$ :

( $\alpha) s_{i}^{p^{n}} \ldots s_{i+t}^{\left(p_{+1}^{n}\right)} \ldots s_{i+p^{n}-1}=1$, for $i \geqslant 2$.

( $\beta$ ) $s_{m+\mu}=1$, for $\mu \geqslant 0$ and $\left[s_{i}, s_{j}\right]=1$ for $i, j \geqslant 2$.

(1.10) For every $i \geqslant 1, H_{i}=\left\langle G_{i}, s\right\rangle$ is of type $(m-i+1, n)$ and has degree of commutativity $i-1$.

(1.11) In the sequel we shall work in metabelian $p$-groups of type $(m, n)$. In this case $G / G_{2}$ acts by conjugation on the abelian group $G_{2}$ and we have

LEMMA. Let $G$ be a metabelian p-group of type $(m+2, n), m>2, \phi$ the natural homomorphism $\phi: \operatorname{Aut}(G) \rightarrow \operatorname{Aut}\left(G_{2}\right)$. Let $s \in G \backslash \Phi(G) G_{1}$ and denote $\alpha=\phi(\bar{s})$. Let $R$ be the subring of $\operatorname{End}\left(G_{2}\right)$ generated by $\alpha$. Then

(a) $G_{2}$ is a cyclic $R$-module, isomorphic to $R$ (as an $R$-module) by $\theta: R \rightarrow G_{2}$, $\theta(r)=s_{2}^{r}$.

(b) $R \cong \mathbf{Z}[t] /\left\langle\left(t^{p^{n}}-1\right) /(t-1),(t-1)^{m}\right\rangle$.

(c) $R$ is a completely primary ring with Jacobson radical $J=\langle\alpha-1, p\rangle$, as the unique maximal ideal of $R$ and $R / J \cong F_{p}$.

(d) The multiplicative group $U$ of the units of $R$ has $1+J$ as a Sylow p-subgroup.

(e) For every subring $K$ of $R$ which lies in $p J, 1+K \cong K$ as abelian groups.

(f) If $H$ is a subring of $J$ such that

$(\alpha) \mho_{1}(1+H) \leqslant 1+p H$ and

( $\beta) ~\left|1+H / \mho_{1}(1+H)\right|=|H / p H|$

then $H \cong 1+H$.

Proof. (a) By (1.9) $G_{2}$ is a cyclic $R$-module generated by $s_{2}$. Since $R \leqslant \operatorname{End}\left(G_{2}\right)$, $G_{2}$ is a faithful $R$-module. Hence $G_{2} \cong R$ as $R$-modules.

(b) Since the defining relations of $G_{2}$ are $\prod_{\mu=0}^{p^{n}-1} s_{i+\mu}^{\left(p_{+1}^{n}\right)}=1$ for $i \geqslant 2$ and $s_{m+2}=1$ by (1.9),

$$
s_{2}^{\sum_{\mu=0}^{p^{n-1}\left(\begin{array}{c}
p_{\mu+1}^{n} \\
n
\end{array}\right)(\alpha-1)^{\mu+j}}}=1
$$


for every $j \geqslant 0$ and by part (a) the defining relations of $R$ are

$$
\sum_{\mu=0}^{p^{n}-1}\left(\begin{array}{c}
p^{n} \\
\mu+1
\end{array}\right)(\alpha-1)^{\mu+j}=0, \quad j \geqslant 0 \text { and }(\alpha-1)^{m}=0 .
$$

Therefore $R \cong \mathbf{Z}[t] / I$ where

$$
I=\left\langle(t-1)^{m}, \sum_{\mu=0}^{p^{n}-1}\left(\begin{array}{c}
p^{n} \\
\mu+1
\end{array}\right)(t-1)^{\mu+j}, j \geqslant 0\right\rangle .
$$

But as

$$
\sum_{\mu=0}^{p^{n}-1}\left(\begin{array}{c}
p^{n} \\
\mu+1
\end{array}\right)(\alpha-1)^{\mu+j}=\alpha^{j} \frac{\alpha^{p^{n}}-1}{\alpha-1},
$$

$I=\left\langle(t-1)^{m},\left(t^{p^{n}}-1\right) /(t-1)\right\rangle$ and the result follows.

(c) and (d) are well-known facts.

(e) It follows by direct calculations that, for $u \in p J, \exp (u)$ and $\ln (1+u)$ defined in the usual manner are isomorphisms from $p J$ to $1+p J$ and from $1+p J$ to $p J$, respectively. (For a more general setting see $[\mathbf{8}]$.)

(f) Since $|1+H|=|H|,(\beta)$ implies that $|1+p H|=|p H|=\left|\mho_{1}(1+H)\right|$. By $(\alpha)$ this means that $\mho_{1}(1+H)=1+p H$. But by part (e) $1+p H \cong p H$, hence $\Omega_{1}(1+H) \cong p H$. Thus $H$ and $1+H$ are two finite abelian $p$-groups with the same number of generators and the same set of invariants. Consequently $H \cong 1+H$ as abelian $p$-groups.

(1.12) Finally, we show that the only nontrivial component of $\operatorname{Aut}(G)$ is its Sylow $p$-subgroup.

Theorem. Let $G$ be a p-group of type $(m, n), m \geqslant 4, p \geqslant 3$. Denote $A=\operatorname{Aut}(G)$ and let $B$ be a Sylow p-subgroup of $A$. Then

(a) $\mid A \| p^{2(m n-2)+1} \cdot(p-1)^{2}$.

(b) $B \triangle A$ and $A$ is a splitting extension of $B$ by a $p^{\prime}$-Hall subgroup $Q$, where $Q$ is isomorphic to a subgroup of $\mathbf{Z}_{p-1} \times \mathbf{Z}_{p-1}$.

(c) $A^{\prime} \leqslant B$.

(d) $A$ is solvable.

(e) $F(A)=B$.

(f) $m-2 \leqslant \operatorname{cl}(B) \leqslant m n-1$.

Proof. We omit the proof of this theorem, as it is straightforward.

2. The structure of the Sylow $p$-subgroup of $\operatorname{Aut}(G)$. It is well known (e.g. [7, Corollary 1]) that if $G$ is a finite $p$-group then $\operatorname{Aut}(G)$ has the following normal series: $1 \triangle K \triangle \operatorname{Aut}(G)$, where $K$ is the set of all the elements of $\operatorname{Aut}(G)$ which fixes $G / K_{2}(G)$ elementwise and $\operatorname{Aut}(G) / K$ is isomorphic to the subgroup of all elements $\operatorname{Aut}(G) / K_{2}(G)$ which can be extended to an automorphism of $G$. Obviously $\bar{G} \triangle K$. In Theorem 2.3 we show that for $p$-groups of type $(m, n), K$ is a splitting extension of $\bar{G}$ by a subgroup of $\operatorname{Aut}(G)$ which fixes a generator of $G$. Also, a lower bound for $|K|$ is given. 
(2.1) Proposition. Let $G$ be a p-group of type $(m, n)$. Let $G_{1}^{\prime} \leqslant G_{l}$ and let $u \in G_{m-l+1} \cap Z\left(G_{1}\right)$, or $u \in G_{2}$ if $G_{2}$ is abelian. Define $\sigma: G \rightarrow G$ by $\sigma: s \rightarrow s, \sigma$ : $s_{1} \rightarrow s_{1} u$ and if $x=s^{b} \prod_{i=1}^{m-1} s_{i}^{a_{i}}, 0 \leqslant b, a_{i}<p^{n}$, then $\sigma: x \rightarrow x \prod_{i=1}^{m-1} u_{i}^{a_{i}}$. Then $\sigma$ is an automorphism of $G$ iff $u_{i}=[u,(i-1) s]$, for $i \geqslant 2$.

Proof. $\sigma$ is a well-defined map of $G$ on itself. We prove, by induction on $|G|$, that $\sigma$ is an automorphism. Let $G_{w}$ be the first abelian $G_{i}$ and denote $H_{w}=\left\langle G_{w}, s\right\rangle$. Then $H_{w}$ is a $p$-group of type $(m-w+1, n)$ by $(1.10)$ and it follows easily from (1.9) that $\sigma_{w}$, the restriction of $\sigma$ to $H_{w}$, is an automorphism of $H_{w}$. Let $H_{2}=\left\langle G_{2}, s\right\rangle$ and assume, by induction, that $\sigma_{2}$ is an automorphism of $H_{2}$. We prove that $\sigma$ is an automorphism of $G$. By induction $\left[s_{i}^{\sigma}, s_{j}^{\sigma}\right]=\left[s_{i}, s_{j}\right]^{\sigma}$ for $i, j \geqslant 2$.

We show that $\left[s_{i}^{\sigma}, s^{\sigma}\right]=s_{i+1}^{\sigma}$ and $\left[s_{i}^{\sigma}, s_{1}^{\sigma}\right]=\left[s_{i}, s_{1}\right]^{\sigma}$. Since $u_{i} \in Z\left(G_{2}\right),\left[s_{i}^{\sigma}, s^{\sigma}\right]=$ $\left[s_{i} u_{i}, s\right]=s_{i+1}\left[u_{i}, s\right]=s_{i+1} u_{i+1}=s_{i+1}^{\sigma}$. Now

$$
\begin{aligned}
{\left[s_{i}^{\sigma}, s_{1}^{\sigma}\right] } & =\left[s_{i} u_{i}, s_{1} u\right]=\left[s_{i}, s_{1} u\right]^{u_{i}}\left[u_{i}, s_{1} u_{1}\right]=\left[s_{i}, u_{1}\right]\left[s_{i}, s_{1}\right]\left[u_{i}, u_{1}\right]\left[u_{i}, s_{1}\right] \\
& =\left[s_{i}, s_{1}\right]\left[u_{i}, s_{1}\right]=\left[s_{i}, s_{1}\right]\left[s_{i}, \sigma, s_{1}\right] .
\end{aligned}
$$

On the other hand $\left[s_{i}, s_{1}\right]^{\sigma}=\left[s_{i}, s_{1}\right]\left[s_{i}, s_{1}, \sigma\right]$. Hence we have to prove

$$
\left[s_{i}, s_{1}, \sigma\right]=\left[s_{i}, \sigma, s_{1}\right] \text {. }
$$

Assume first that $G_{2}$ is not abelian. Then by assumption $\left[s_{i}, s_{1}, \sigma\right] \leqslant\left[G_{1}^{\prime}, \sigma\right] \leqslant$ $G_{l+m-l}=G_{m}=1$. So

$$
\left[s_{i}, s_{1}, \sigma\right]=1 \text {. }
$$

On the other hand, if $x \in Z\left(G_{1}\right)$, then $[x, s] \in Z\left(G_{1}\right)$. Consequently $\left[u_{i}, s_{1}\right]=1$ for $i>1$ and

$$
\left[s_{i}, \sigma, s_{1}\right]=1
$$

(1) and (2) imply (*).

Assume now that $G_{2}$ is abelian. Let notation be as in Lemma 1.11 and denote by $\sigma_{2}$ the restriction of $\sigma$ to $G_{2}$. Then $\sigma_{2} \in R$, by the definition of $\sigma$. Since $s_{i}$, $\left[s_{i}, s_{1}\right] \in G_{2}$, Lemma 1.11(b) implies $\left[s_{i}, s_{1}, \sigma\right]=\left[s_{i}, \phi\left(s_{1}\right), \sigma_{2}\right]=s_{i}^{f(\alpha) g(\alpha)}$, where $f(t), g(t) \in \mathbf{Z}[t]$, and $\left[s_{i}, \sigma, s_{1}\right]=\left[s_{i}, \sigma_{2}, \phi\left(s_{1}\right)\right]=s_{i}^{g(\alpha) f(\alpha)}$. Since $R$ is commutative, $(*)$ holds.

Finally, if $v \in G_{1} \backslash G_{2} \Phi\left(G_{1}\right)$ then by the collection formula

$$
(s v)^{p^{n}}=s^{p^{n}} v^{p^{n}} \prod_{i} d_{i}(s, v)
$$

where $d_{i}(s, v)$ are certain commutators in $s$ and $v$. If $v_{1}=v^{\sigma}$, then since $d_{i}(s, v), s^{p^{n}}$, $v^{p^{n}} \in G_{2}$,

$$
\left\{\begin{array}{l}
\left((s v)^{\sigma}\right)^{p^{n}}=\left(s v_{1}\right)^{p^{n}}=s^{p^{n}} v_{1}^{p^{n}} \prod_{i} d_{i}\left(s, v_{1}\right)=s^{p^{n}} v_{1}^{p^{n}} \prod_{i} d_{i}\left(s, v^{\sigma}\right) \\
\left((s v)^{p^{n}}\right)^{\sigma}=\left(s^{p^{n}} v^{p^{n}} \prod_{i} d_{i}(s, v)\right)^{\sigma}=\left(s^{p^{n}}\right)^{\sigma}\left(v^{p^{n}}\right)^{\sigma} \prod_{i} d_{i}\left(s, v^{\sigma}\right)
\end{array}\right.
$$

Since $[v, \sigma]=\tilde{u} \in G_{2}, \quad((s v) \sigma)^{p^{n}}=(s v \tilde{u})^{p^{n}}=(s v)^{p^{n}}$ and, as $(s v)^{p^{n}} \notin Z(G)$, $\left((s v)^{p^{n}}\right)^{\sigma}=(s v)^{p^{n}}$. Hence $\left((s v)^{\sigma}\right)^{p^{n}}=\left((s v)^{p^{n}}\right)^{\sigma}$. But then by (4) $\left(v^{p^{n}}\right)^{\sigma}=\left(v^{\sigma}\right)^{p^{n}}$ 
and since $G_{1} / G_{2}$ is cyclic, this proves that $\sigma \in \operatorname{Aut}(G)$. The other direction follows from Witt's identity with $a=s_{1}, b=s^{-1}$ and $c=\sigma$ in formula $(\delta)$ of $\S 0$.

(2.2) Proposition. Let $G$ be a finite p-group of type $(m, n), m \geqslant 4$. Then to every $u \in G_{2}$ there exists a solution of the equation $[s, x] u[u, x]=1$ in $x \in G_{1}$.

Proof. We have to prove $u^{x}=[x, s]$, for some $x \in G_{1}$. By (1.3) $u=\left[s, x^{-1}\right]$ for some $x \in G_{1}$. So $u^{x}=\left[s, x^{-1}\right]^{x}=[s, x]^{-x^{-1} \cdot x}=[x, s]$, by $0(\alpha)$.

I am indebted to the referee for this short proof.

(2.3) TheOrem. Let $G$ be a p-group of type $(m, n), m \geqslant 4$, and let $P$ be the Sylow p-subgroup of $\operatorname{Aut}(G)$.

Let $A_{3}=\left\{\sigma \in \operatorname{Aut}(G) \mid[s, \sigma]=1,\left[s_{1}, \sigma\right] \in G_{3}\right\}$ and let $B$ be the subgroup of $\operatorname{Aut}(G)$ which fixes $G / G_{2}$ elementwise. Then

(a) $\left|A_{3}\right| \geqslant\left|G_{m-1+1} \cap Z\left(G_{1}\right)\right|$, where $G_{1}^{\prime} \leqslant G_{l}$ but $G_{1}^{\prime} \$ G_{l-1}$.

(b) $B$ is a splitting extension of $\bar{G}$ by $A_{3}$.

Proof. (a) follows from Proposition 2.1.

(b) It follows from the definitions of $A_{3}$ and $\bar{G}$ that $A_{3} \cap \bar{G}=\{1\}$. Hence it remains to show that $A_{3} \bar{G}=B$. Obviously $A_{3} \bar{G} \leqslant B$. Let $\sigma \in B,[s, \sigma]=u,\left[s_{1}, \sigma\right]=$ $v, u, v \in G_{2}$. By Proposition 2.2 there is an element $x \in G_{1}$ such that $[s, x] u[u, x]$ $=1$. Hence $s^{\sigma x}=(s u)^{x}=s[s, x] u[u, x]=s$ and $s_{1}^{\sigma x}=s_{1} v_{1}$, where $v_{1}=$ $\left[s_{1}, x\right] v[v, x] \in G_{2}$. Assume that $v_{1} \equiv s_{2}^{\alpha} \bmod G_{3}, 0 \leqslant \alpha<p^{n}$. Then $\sigma \bar{x} \bar{s}^{-\alpha}: s \rightarrow s$ and $\sigma \bar{x} \bar{s}^{-\alpha}: s_{1} \rightarrow\left[s_{1}, v_{1}\right]^{s(-\alpha)} \equiv s_{1} s_{2}^{-\alpha} v_{1}\left[v_{1}, s^{-\alpha}\right] \equiv s_{1} s_{2}^{-\alpha} s_{2} \equiv \bmod G_{3}$, i.e. $\sigma \bar{x} \bar{s}^{-\alpha} \in$ $A_{3}$. Therefore $\sigma \in A_{3} \bar{G}$. Consequently $B=A_{3} \bar{G}$, as required.

COROLlaRY. Let notation be as in the theorem. If $G$ has degree of commutativity $l$ then $|\operatorname{Aut}(G) / \bar{G}| \geqslant p^{n t}$, where $t=\min \{m-l-1, l+3\}$.

3. Metabelian $p$-groups of type $(m, n)$. To prove the main result of this section (Theorem 3.2) we need the following:

(3.1) Lemma. Let $G, R$ and $\phi$ be as defined in Lemma 1.11. For every $i \geqslant 3$ let $A_{i}=\left\{\alpha \in \operatorname{Aut}(G) \mid[s, \alpha]=1,\left[s_{1}, \alpha\right] \in G_{i}\right\}$ and let $B=\bar{G} A_{3}$ as in Theorem 2.3. Assume that $G$ has an automorphism $\tau$ such that $s^{\tau}=s s_{1}^{-1}$ and $s_{1}^{\tau} \equiv s_{1} \bmod G_{3}$ and which induces an automorphism on $R$ such that $x^{\tau}=x+y+x y$, where $x=\phi(s)-1$ and $y=\phi\left(\bar{s}_{1}^{-1}\right)-1$. Then for every $i \geqslant 3$

(a) $\phi\left(A_{i}\right)=1+x^{i-1} R$.

(b) If $Z\left(G_{1}\right)=G_{m-k}$ then $C_{G_{2}}\left(\left[1+x^{i-1}, \tau\right]\right) \geqslant G_{m-k-i+2}, C_{G_{2}}\left(\left[1+x^{i-1}, \tau\right]\right) \neq$ $G_{m-k-i+1}$ and

(c) $\left[1+x^{i-1}, \tau\right] \in 1+x^{i+k-2} R \backslash 1+x^{i+k-1} R$.

(d) If $\alpha \in A_{i} \backslash A_{i+1}$ then $[\tau, \alpha] \in \bar{G}_{i-1} A_{i+k-1} \backslash \bar{G}_{i-1} A_{i+k}$, for $i \leqslant m-k$ and $[\tau, \alpha]$ $\in \bar{G}_{i-1}$, for $i>m-k$.

Proof. (a) Let $\alpha \in A_{i}$. Then by Proposition 2.1 there exists a $u \in G_{i+1}$ such that $\left[s_{2}, \alpha\right]=u$. Since $G_{2}$ is a cyclic $R$-module by Lemma 1.11(a), there exists a polynomial $f(t) \in \mathbf{Z}[t] t^{i-1}$ such that $u=s_{2}^{f(x)}$. We claim that $\phi(\alpha)=1+f(x)$. Since $1+f(x)$ and $\phi(\alpha)$ are $R$-endomorphisms of $G_{2}$, it suffices to show that 
$s_{2}^{\phi(\alpha)}=s_{2}^{1+f(x)}$. But $s_{2}^{\phi(\alpha)}=s_{2}^{\alpha}=s_{2} u=s_{2} \cdot s_{2}^{f(x)}=s_{2}^{1+f(x)}$. Hence $\phi(\alpha)=1+f(x)$ and $\phi\left(A_{i}\right) \subseteq 1+x^{i-1} R$. Conversely, let $f(t) \in \mathbf{Z}[t] t^{i-1}$ and let $u=s_{2}^{f(x)}$. Then $u \in G_{i+1}$ and $s_{2}^{1+f(x)}=s_{2} u$. Since for every $u \in G_{i+1}$ there exists an $\alpha \in A_{i}$ such that $s_{2}^{\alpha}=s_{2} u$ by Proposition 2.1, $1+f(x)=\phi(\alpha)$ for some $\alpha \in A_{i}$. Consequently, $\phi\left(A_{i}\right)=1+x^{i-1} R$.

(b) It suffices to show that $j=m-k-i+2$ is the smallest $j$ such that $s_{j}^{\left[1+x^{i-1}, \tau\right]}$ $=s_{s}$. Denote $\sigma=1+x^{i-1}$ for brevity. Then since $\sigma^{\tau} \in R$, by definition, $[\sigma, \tau]=$ $\boldsymbol{\sigma}^{-1} \boldsymbol{\sigma}^{\tau}=\boldsymbol{\sigma}^{\tau} \boldsymbol{\sigma}^{-1}$, as $R$ is commutative. Hence $s_{j}^{[\boldsymbol{\sigma}, \tau]}=s_{j} \Leftrightarrow s_{j}^{[\boldsymbol{\sigma}, \tau]-1}=1 \Leftrightarrow s_{j}^{\boldsymbol{\sigma}^{-1} \boldsymbol{\sigma}^{\tau-1}}=$ $1 \Leftrightarrow s_{j}^{\left(\sigma^{\tau} \sigma^{-1}-1\right) \sigma}=1 \Leftrightarrow s_{j}^{\sigma^{\tau}-\sigma}=1$, i.e. $s_{j}^{[\sigma, \tau]}=s_{j} \Leftrightarrow s_{j}^{\boldsymbol{\sigma}^{\tau}-\sigma}=1$. Now

$$
\sigma^{\tau}-\sigma=(x+y+x y)^{i-1}-x^{i-1}=g(x, y)
$$

and $g(x, y)=y(x-1) \sum_{\mu=0}^{i-2} x^{i-2-\mu}(x+y+x y)^{\mu}$.

To every $j \geqslant 2 s_{j}^{x^{a} y^{b}}=\left[s_{j+a}, b s_{1}\right], a, b \in \mathbf{Z}$. Therefore, if $\left[s_{1}, s_{2}\right] \equiv s_{r}^{\delta} \bmod G_{r+1}$ and $(\delta, p)=1$ then $s_{j}^{x^{a} y^{b}} \equiv s_{b(r-2)+j+a}^{\varepsilon} \bmod G_{b(r-2)+j+a+1},(\varepsilon, p)=1$, by 1.9(b). Hence if $g(x, y)=\Sigma c_{a, b} x^{a} y^{b}$ and $b(r-2)+j+a$ attains its minimum for a unique pair $(a, b)$ such that $c_{a, b} \not o(p)$, then $s_{j}^{g(x, y)}=s_{j}$ iff $s_{j}^{x^{a} y^{b}}=s_{j}$. But in $g(x, y)$ of $(*), b(r-2)+j+a$ obtains its minimal value for $a=i-2$ and $b=1$, as $r \geqslant 4$ by the definition of $G_{1}$, and for this $(a, b), c_{a, b}=-1$. Therefore $s_{j}^{[\sigma, \tau]}=s_{j}$ iff $\left[s_{j+i-2}, s_{1}\right]=1$, i.e. $s_{j+i-2} \in Z\left(G_{1}\right)$. Thus $s_{j+i-2} \in G_{m-k}, j+i-2 \geqslant m-k$ and $j \geqslant m-k-i+2$. By the choice of $j, j=m-k-i+2$. Hence $G_{m-k-i+2} \subseteq$ $C_{G_{2}}\left(\left[1+x^{i-1}, \tau\right]\right)$ and $G_{m-k-i+1} \nsubseteq C_{G_{2}}\left(\left[1+x^{i-1}, \tau\right]\right)$, as required.

(c) If $\left[1+x^{i-1}, \tau\right] \in 1+x^{l} R \backslash 1+x^{l+1} R$ then the smallest $j$ such that $s_{j}^{\left[1+x^{i-1}, \tau\right]}$ $=s_{j}$ is $j=m-l$. Hence by part (b) $m-k-i+2=m-l$, i.e. $l=k+i-2$, as required.

(d) We prove (d) in four steps.

Step I. $[\alpha, \tau] \in \bar{G}_{2} A_{3}$.

To prove this it suffices to show that $s^{[\alpha, \tau]} \equiv s \bmod G_{3}$ and $s_{1}^{[\alpha, \tau]} \equiv s_{1} \bmod G_{3}$.

$$
\begin{aligned}
s^{\alpha \tau \alpha^{-1} \tau^{-1}}=s^{\tau \alpha^{-1} \tau^{-1}} & =\left(s s_{1}^{-1}\right)^{\alpha^{-1} \tau^{-1}}=\left(s s_{1}^{-1}\left[s_{1}^{-1}, \alpha^{-1}\right]\right)^{\tau^{-1}} \\
& =s\left[s, \tau^{-1}\right] s_{1}^{-\tau^{-1}}\left[s_{1}^{-1} \alpha^{-1}\right]^{\tau^{-1}} .
\end{aligned}
$$

Since $\left[s, \tau^{-1}\right]=[s, \tau]^{-\tau^{-1}}=s_{1}^{\tau^{-1}}$ we obtain

$$
s^{\alpha \tau \alpha^{-1} \tau^{-1}}=s\left[s_{1}^{-1}, \alpha^{-1}\right]^{-1} \equiv s \bmod G_{i}, \quad i \text { defined by assumption. }
$$

In particular $s^{\alpha \tau \alpha^{-1} \tau^{-1}} \equiv s \bmod G_{3}$. Clearly $s_{1}^{\alpha \tau \alpha^{-1} \tau^{-1}} \equiv s_{1} \bmod G_{3}$. This proves Step I. Step II. $[\alpha, \tau] \in \bar{G}_{2} A_{i+k-1} A_{m-1} \backslash \bar{G}_{2} A_{i+k} A_{m-1}$ for $i+k \leqslant m-1$ and $[\alpha, \tau] \in$ $\bar{G}_{2} A_{i+k-1} A_{m-1}$ for $i+k>m-1$. Let $\tau \in \operatorname{Aut}(G)$ satisfying $[s, \tau]=s_{1}^{-1},\left[s_{1}, \tau\right] \in$ $G_{3}$. We show that $\tau$ induces an automorphism on $R$ by

$$
\tau: \sum a_{i} x^{i} \rightarrow \sum a_{i}(x+y+x y)^{i} .
$$

Here $x$ and $y$ are as defined in the lemma. Obviously $\tau$ maps $R$ onto itself; hence by Lemma 1.11(b) it suffices to show that if $y=f(x), f(t) \in \mathbf{Z}[t]$, then

$$
t+f(t)+t f(t) \in I \quad \text { and } \quad \sum_{i=1}^{p^{n}}\left(\begin{array}{l}
p^{n} \\
i
\end{array}\right)(t+f(t)+t f(t))^{i-1} \in I .
$$


Here $I=\left\langle t^{m},\left((1+t)^{p^{n}}-1\right) / t\right\rangle$ and we have written $t$ instead of $t-1$ in Lemma 1.11(b). As $f(t) \in t^{2} R$, by the definition of $s_{1}, t+f(t)+t f(t) \in t R$ and $(t+f(t)+t f(t))^{m} \in t^{m} R \leqslant I$. Finally let $\tilde{s}_{i}=\left[s_{1},(i-1) s s_{1}^{-1}\right]$ for $i \geqslant 2$. As $s s_{1}^{-1} \in$ $G \backslash G_{1} \Phi(G)$,

$$
\tilde{s}_{2}^{p^{n}} \tilde{s}_{3}^{\left(\xi^{n}\right)} \ldots \tilde{s}_{j}^{\left(\rho_{j}^{n}\right)} \ldots \tilde{s}_{p^{n}+1}=1
$$

by $1.9(\alpha)$. Thus, if $R_{1}$ is the subring of End $G_{2}$ generated by $\phi\left(\overline{s s}_{1}^{-1}\right)$, then $G_{2}$ is a faithful cyclic $R_{1}$ - module generated by $\tilde{s}_{2}$ and

$$
\tilde{s}_{2}^{p^{n}} \tilde{s}_{3}^{\left(2^{n}\right)} \tilde{s}_{j}^{\left(p_{j}^{n}\right)} \ldots \tilde{s}_{p^{n}+1}=1
$$

implies that

$$
\sum_{i=1}^{n}\left(\begin{array}{c}
p^{n} \\
i
\end{array}\right)\left(\phi\left(\overline{s s}_{1}^{-1}\right)-1\right)^{i-1}=0 \quad \text { in } R
$$

Hence

$$
\begin{aligned}
\left(\sum_{i=1}^{p^{n}}\left(\begin{array}{c}
p^{n} \\
i
\end{array}\right) x^{i-1}\right)^{\tau} & =\sum_{i=1}^{p^{n}}\left(\begin{array}{c}
p^{n} \\
i
\end{array}\right)(x+y+x y)^{i-1} \\
& =\sum_{i=1}^{p^{n}}\left(\begin{array}{c}
p^{n} \\
i
\end{array}\right)((x+1)(y+1)-1)^{i-1}=0
\end{aligned}
$$

and $\sum_{i=1}^{p^{n}}\left({ }_{i}^{p^{n}}\right)(x+y+x y)^{i-1}=0$. Therefore by Lemma 1.11(b) the natural homomorphism $\theta: Z[t] \rightarrow Z[t] / I$ sends $\sum_{i=1}^{p^{n}}\left({ }_{i}^{p^{n}}\right)(t+f(t)+t f(t))^{i-1}$ to the zero element of $Z[t] / I$ and $I^{\tau}=I$. Thus, since $\tau$ induces a homomorphism on $Z[t]$, it induces an automorphism on $Z[t] / I$ and consequently on $R$. We claim that $\phi([\alpha, \tau]) \in$ $x^{i+k-2} R \backslash x^{i+k-1} R$. Indeed, as $\tau$ induces an automorphism on $R,\left[1+x^{i-1}, \tau\right] \in 1$ $+x^{i+k-2} R \backslash 1+x^{i+k-1} R$ by part (c) and, for every $r \in R \backslash x R,\left[1+x^{i-1}, \tau\right] \in 1$ $+x^{i+k-1} R$. (The last assertion follows by induction on $m-\operatorname{deg} f(t)$, where $f(x)=$ $r, f(t) \in Z[t]$.) But by the definition of $\tau, \phi([\alpha, \tau])=[\phi(\alpha), \tau]$. Consequently $\phi([\alpha, \tau])=\left[1+x^{i-1} r, \tau\right] \in 1+x^{i+k-2} R \backslash 1+x^{i+k-1} R$ by parts (a) and (c) and $[\alpha, \tau] \in \phi^{-1}\left(1+x^{i+k-2} R\right) \backslash \phi^{-1}\left(1+x^{i+k-1} R\right)=\bar{G}_{2} A_{i+k-1} A_{m-1} \backslash \bar{G}_{2} A_{i+k} A_{m-1}$ for $i+k \leqslant m-1$ and $[\alpha, \tau] \in \bar{G}_{2} A_{i+k-1} A_{m-1} \backslash \bar{G}_{2} A_{i+k} A_{m-1}$.

Step III. $[\alpha, \tau] \in \bar{G}_{i-1} A_{i+k-1} A_{m-1}$. Let $[\alpha, \tau]=\beta \bar{g}, \bar{g} \in \bar{G}_{2}, \beta \in A_{i+k-1} A_{m-1}$. Then $s^{[\alpha, \tau]}=s^{\beta \bar{g}}=s^{\bar{g}}$, as $s^{\beta}=s$. By (1) $s^{[\alpha, \tau]} \equiv s \bmod G_{i}$. Hence $s^{\bar{g}} \equiv s \bmod G_{i}$ and this means that $[s, g] \in G_{i}$. Consequently $g \in G_{i-1}$.

Step IV. $[\alpha, \tau] \in \bar{G}_{i-1} A_{i+k-1} \backslash \bar{G}_{i-1} A_{i+k}$ for $i \leqslant m-k$ and $[\alpha, \tau] \in \bar{G}_{i-1}$ for $i \geqslant m-k+1$. If $i+k-1 \leqslant m-1$ then $A_{i+k-1} \geqslant A_{m-1}$ and nothing has to be proved, by Step III. Hence assume $i+k \geqslant m+1$, i.e. $i \geqslant m-k+1$. We show that $\left[A_{m-k+1}, \tau\right] \leqslant \bar{G}_{2}$. For this it suffices to show that if $\alpha \in A_{m-k+1}$ then $s_{1}^{[\alpha, \tau]}=s_{1}$; for $[\alpha, \tau]=\bar{g}^{\beta}, \bar{g} \in \bar{G}_{m-k}$, and $\beta \in A_{m-1}$ by Step III. Hence $\beta=1 \Leftrightarrow s_{1}^{\beta}=s_{1} \Leftrightarrow$ $s_{1}^{[\alpha, \tau]}=s_{1}$, as $g \in G_{m-k}=Z\left(G_{1}\right)$. Let $\left[s_{1}, \alpha\right]=v$ and $\left[s_{1}, \tau\right]=u$. It follows by induction on $j$ that $\left[s_{j}, \tau\right]=[u,(j-1) s] \cdot \Pi\left[x_{1}, \ldots, x_{\mu}\right]$ where $x_{h} \in\left\{s, u, s_{r}, 1 \leqslant r\right.$ $\leqslant j\}, \mu \geqslant j$, and at least two of the $x_{h}$ 's differ from $s$. Since $G$ is metabelian, if $\left[x_{1}, \ldots, x_{\mu}\right] \neq 1$ then at most one of the $x_{h}$ is an element of $G_{2}$. Hence at least one of 
the $x_{h}$ is $s_{1}$ and as $G$ is metabelian, we may assume $x_{\mu}=s_{1}$. But if $\mu \geqslant m-k+1$ then $\left[x_{1}, \ldots, x_{\mu-1}\right] \in G_{m-k}=Z\left(G_{1}\right)$; consequently $\left[x_{1}, \ldots, x_{\mu}\right]=1$. Therefore, $\left[s_{j}, \tau\right]=[u,(j-1) s]$ for $j \geqslant m-k+1$. Consequently, $[v, \tau]=[u, \alpha]=s_{2}^{f(x) g(x)}$, where $f(t), g(t) \in \mathbf{Z}[t], v=s_{2}^{f(x)}, u=s_{2}^{g(x)}$ and $x=\phi(\bar{s})-1$. This implies that $s_{1}^{\alpha \tau}=\left(s_{1} v\right)^{\tau}=s_{1} u \cdot v[v, \tau]=s_{1} v u[u, \alpha]=\left(s_{1} u\right)^{\alpha}=s_{1}^{\tau \alpha}$ and $s_{1}^{[\alpha, \tau]}=s_{1}$, as required.

(3.2) THEOREM. Let $G$ be a metabelian p-group of type $(m, n), m \geqslant 4$, and for every $i \geqslant 3$ let $A_{i}=\left\{\sigma \in \operatorname{Aut}(G) \mid[s, \sigma]=1\right.$ and $\left.\left[s_{1}, \sigma\right] \in G_{i}\right\}, A=\{\sigma \in \operatorname{Aut}(G) \mid[s, \sigma]$ $=1\}$. Then
(a) $A=A_{3} \times\langle\bar{s}\rangle$ is abelian.
(b) $\left|A_{3}\right|=\left|G_{3}\right|$.
(c) Let $H \leqslant \mho\left(G_{3}\right) \mho_{2}\left(G_{2}\right)$ such that $H^{s}=H$ and let $A_{H}=\left\{\sigma \in A \mid\left[s_{2}, \sigma\right] \in H\right\}$. Then $A_{H} / A_{H} \cap A_{m-1} \cong H$.

(d) The Sylow p-subgroup $P$ of $\operatorname{Aut}(G)$ is generated by $p^{n}+4$ elements.

(e) $K_{i}(B)=\bar{G}_{i}$ and $Z_{i}(B)=\bar{G}_{m-i-1} A_{m-1}$. Here $B=\bar{G} \cdot A_{3}$.

(f) Assume that $G$ can be embedded in a p-group $G_{0}$ of type $(m+1, n)$ and let $B_{0}$ be the set of all the elements of $\operatorname{Aut}\left(G_{0}\right)$ which fix $G_{0} / K_{2}\left(G_{0}\right)$ elementwise. If $Z\left(G_{1}\right)=$ $G_{m-k}$ then $A_{(i-1) \cdot(k-1)+2} \bar{G}_{i-1}<K_{i}\left(B_{0}\right) \leqslant A_{(i-1)(k-1)+3} \cdot \bar{G}_{i-1}$ and

(g) $Z_{i}\left(B_{0}\right)=A_{m-i-1} \bar{G}_{m-i+1}$.

Proof. (a) $A=A_{3} \times\langle\bar{s}\rangle$ by the definitions of $A, A_{3}$ and by Theorem 2.3. Hence we show that $A_{3}$ is abelian. Let $\alpha, \beta \in A_{3},\left[s_{1} \alpha\right]=u,\left[s_{1}, \beta\right]=v$. Then $s_{1}^{\alpha \beta}=\left(s_{1} u\right)^{\beta}$ $=s_{1} v u[u, \beta]$ and $s_{1}^{\beta \alpha}=\left(s_{1} v\right)^{\alpha}=s_{1} u v[v, \alpha]$. Hence $s_{1}^{\alpha \beta}=s_{1}^{\beta \alpha}$ iff $[v, \alpha]=[u, \beta]$. We show $[v, \alpha]=[u, \beta]$. Let $R$ be the ring defined in Lemma 1.11; denote $x=\phi(\bar{s})-1$, where $\phi$ is the canonical homomorphism from $\operatorname{Aut}(G)$ to $\operatorname{Aut}\left(G_{2}\right)$. Then for every element $a \in G_{2}$ there exists a polynomial $f_{0}(t) \in \mathbf{Z}[t]$ such that $a=s_{2} f_{0}(x)$. In particular $v=s_{2}^{f(x)}, u=s_{2}^{g(x)}$ for suitable $f(t), g(t) \in \mathbf{Z}[t]$. Now $[u, \beta]=[u, \phi(\beta)]$ $=s_{2}^{g(x)(\phi(\beta)-1)}=s_{2}^{g(x) f(x)}=s_{2}^{f(x) g(x)}=v^{g(x)}=v^{(\phi(\alpha)-1)}=[v, \alpha]$, as in the proof of Lemma 3.1(a).

(b) Follows from Theorem 2.3(a).

(c) Let notation be as in Lemma 1.11. Then $\theta(p J)=\mho_{1}\left(G_{3}\right) \cdot \mho_{2}\left(G_{2}\right)$. Hence if $H \leqslant \mho_{1}\left(G_{3}\right) \cdot \mho_{2}\left(G_{2}\right)$ then $\theta^{-1}(H) \subseteq 1+p J$ and, as $H$ is $s$-invariant, $\theta^{-1}(H) \cong 1+$ $\theta^{-1}(H)$ by Lemma 1.11(c). But $1+\theta^{-1}(H)=\phi\left(A_{H}\right)$. Hence $A_{H} / \operatorname{Ker} \phi \cap A_{H} \cong 1$ $+\theta^{-1}(H) \cong \theta^{-1}(H) \cong H$ and $H \cong A_{H} / A_{H} \cap A_{m-1}$ as $\operatorname{Ker} \phi=\bar{G}_{2} A_{m-1}$ and $A_{H} \leqslant$ A.

(d) It is not difficult to see that $A_{3}$ is generated by $\left\{\sigma_{i} \mid \sigma_{i}: s_{1} \rightarrow s_{1} s_{i}, 3 \leqslant i \leqslant p^{n}+\right.$ 2). Hence $A_{3}$ is generated by $p^{n}-1$ elements and $B=\bar{G} A_{3}$ is generated by $p^{n}+1$ elements. Every $p$-subgroup of $\mathrm{GL}\left(2, \mathbf{Z}_{p^{n}}\right)$ can be generated by 3 elements. Hence $P$ is generated by $p^{n}+4$ elements.

(e) By Theorem 2.3(b) $B / \bar{G}_{1} \cong A$ and by part (a) of Theorem $3.2 A$ is abelian. Hence $K_{2}(B) \leqslant \bar{G}_{1}$. On the other hand $\left[\phi\left(\bar{s}_{1}\right), \phi(A)\right]=1$, i.e. $\left[\bar{s}_{1}, A\right] \leqslant \bar{G}_{2} A_{m-1}$. Therefore as $A$ is abelian, $K_{2}(B)=[B, B]=\left[\bar{G}_{1} A, \bar{G}_{1} A\right] \leqslant \bar{G}_{2}\left[\bar{G}_{1}, A\right] \leqslant \bar{G}_{1} \cap$ $\bar{G}_{2} A_{m-1}=\bar{G}_{2}$. But obviously $\bar{G}_{2} \leqslant K_{2}(B)$. Consequently $K_{2}(B)=\bar{G}_{2}$. Since $\left[\bar{G}_{i}, \bar{s}\right]$ $=\bar{G}_{i+1}$ for $i \geqslant 2$, we get by induction on $i$ that $K_{i}(B)=\bar{G}_{i}$ for $2 \leqslant i \leqslant m-2$. To determine the upper central series of $B$ determine first $Z(B)$. Let $\sigma \in Z(B), \sigma=\bar{g} \rho$, 
$\bar{g} \in \bar{G}, \rho \in A_{3}$. Since $[\bar{s}, \sigma]=[\bar{s}, \bar{g}]^{\rho},[\bar{s}, \bar{g}]=1$ and $g \in G_{m-2}$. Also, as $G$ has degree of commutativity $\geqslant 1$ by $(1.8)$ and $\bar{g} \in \bar{G}_{m-2},\left[\bar{s}_{1}, \sigma\right]=\left[\bar{s}_{1}, \rho\right]$ and $\left[\bar{s}_{1}, \rho\right]=1$. This implies that $\left[s_{1}, \rho\right] \in G_{m-1}$. Consequently $\sigma \in \bar{G}_{m-2} A_{m-1}$ and $Z(B) \leqslant$ $\bar{G}_{m-2} A_{m-1}$. But obviously $\bar{G}_{m-2} A_{m-1} \leqslant Z(B)$. Thus $Z(B)=\bar{G}_{m-2} A_{m-1}$. Since $Z(B)$ is the kernel of the natural homomorphism $\psi: \operatorname{Aut}(G) \rightarrow \operatorname{Aut}\left(G / G_{m-1}\right)$, we get the results by induction on $\operatorname{cl}(G)$.

(f) Since $G$ may be embedded in $G_{0}$ there exists a $\tau \in \operatorname{Aut}(G)$ such that $s^{\tau}=s s_{1}^{-1}$ ( $\tau$ plays here the role of $s_{1}$ in $G$ ). Since $\tau \notin B$ and $B \triangle \operatorname{Aut}(G)$ by Theorem 2.3(b), $\tau$ acts by conjugation on $B$ and

$$
B_{0}=B\langle\tau\rangle, \quad[\bar{s}, \tau]=\bar{s}_{1} \quad \text { and } \quad\left[\bar{s}_{1}, \tau\right] \in G_{3} .
$$

We compute $K_{2}\left(B_{0}\right)$ and then $K_{i}\left(B_{0}\right)$ for $i \geqslant 3$ by induction on $i$. Since $B_{0} / B$ is cyclic by $(2), K_{2}\left(B_{0}\right)=\left[B_{0}, B\right]=\left[B, A_{3}\right]^{\tau}[B, \bar{G}]^{\tau}\left[\tau, A_{3}\right] \cdot[\tau, \bar{G}]^{A_{3}} \leqslant \bar{G}_{1}\left[\tau, A_{3}\right]$. By Lemma $3.1(\mathrm{~d})\left[\tau, A_{3}\right] \leqslant \bar{G}_{2} A_{k+2}$. Hence $K_{2}\left(B_{0}\right) \leqslant \bar{G}_{1} A_{k+2}$. Since $[\bar{s}, \tau]=\bar{s}_{1}^{-1}, \bar{G}_{1}$ $\leqslant K_{2}\left(B_{0}\right)$. Now

$$
\begin{aligned}
{\left[\bar{G}_{i} A_{j}, B_{0}\right] } & =\left[\bar{G}_{i}, B_{0}\right]\left[A_{j}, B_{0}\right]=\left[A_{j}, B_{0}\right] \bar{G}_{i+1}=G_{i+1}\left[A_{j},\langle\tau\rangle B\right] \\
& =\bar{G}_{i+1}\left[A_{j}, B\right]\left[A_{j}, \tau\right]\left[A_{j}, \tau, B\right] \leqslant \bar{G}_{i+1} \bar{G}_{j} A_{j+k-1} \backslash \bar{G}_{i+1} \bar{G}_{j} A_{j+k}
\end{aligned}
$$

by Lemma 3.1(d). Therefore,

$$
K_{i+1}\left(B_{0}\right)=\left[K_{i}\left(B_{0}\right), B_{0}\right]=\left[\bar{G}_{i-1} A_{3+(i-1)(k-1)}, B_{0}\right] \leqslant \bar{G}_{i} A_{3+i(k-1)} \backslash \bar{G}_{i} A_{2+i(k-1)} .
$$

Also, $\bar{G}_{i} \leqslant K_{i+1}\left(B_{0}\right)$, as $[\tau, i \bar{s}] \in K_{i+1}\left(B_{0}\right)$.

(h) First we compute $Z\left(B_{0}\right)$. Obviously $Z\left(B_{0}\right) \leqslant Z(B)$ as $Z\left(B_{0}\right) \leqslant B_{0}$. Hence $Z\left(B_{0}\right) \leqslant A_{m-1} \bar{G}_{m-2}$. We show that $Z\left(B_{0}\right)=\bar{G}_{m-2}$. Let $\sigma \in A_{m-1} \cap Z\left(B_{0}\right)$. Then $\left[s_{1}, \sigma\right] \in G_{m-1}$ and if $[s, \sigma]=z$ then $s=s^{\sigma \tau \sigma^{-1} \tau^{-1}}=s^{\tau \sigma^{-1} \tau^{-1}}=\left(s s_{1}^{-1}\right)^{\sigma^{-1} \tau^{-1}}=$ $\left(s s_{1}^{-1}\left[s_{1}^{-1}, \sigma^{-1}\right]\right)^{\tau^{-1}}=s\left[s_{1}^{-1}, \sigma^{-1}\right]^{\tau^{-1}}=s z$. Hence $z=1$ and $\left[s_{1}, \sigma\right]=1$, i.e. $\sigma=1$. On the other hand $\bar{s}_{m-2} \in Z\left(B_{0}\right)$ as $s^{\bar{s}_{m-2^{T}} \bar{s}_{m-2} \tau^{-1}}=s$ and $s_{1}^{\bar{s}_{m-2} \bar{s}_{m-2}^{-1} \tau^{-1}}=s_{1}$. Consequently $Z\left(B_{0}\right)=\bar{G}_{m-2}$. Next we compute $Z_{2}\left(B_{0}\right)$. Let $\psi: \operatorname{Aut}(G) \rightarrow \operatorname{Aut}\left(G / G_{m-1}\right)$ be the natural homomorphism and let $B_{1}=\psi\left(B_{0}\right)$. Then Ker $\psi=\bar{G}_{m-2} A_{m-1}$ and $\operatorname{Ker} \psi \leqslant Z_{2}\left(B_{0}\right) \leqslant \psi^{-1}\left(Z\left(B_{1}\right)\right)$. For, by Lemma 3.1(d) if $\sigma \in A_{m-1}$ then $[\sigma, \tau] \in$ $\bar{G}_{m-2}=Z\left(B_{0}\right)$; hence $\operatorname{Ker} \psi=\bar{G}_{m-2} A_{m-1} \leqslant Z_{2}\left(B_{0}\right)$. Also $Z_{2}\left(B_{0}\right)=\left\{\sigma \in B_{0} \mid\right.$ $[\sigma, \rho] \in \bar{G}_{m-2}$ for every $\left.\rho \in B_{0}\right\} \leqslant\left\{\sigma \in B_{0} \mid[\sigma, \rho] \in \bar{G}_{m-2} A_{m-1}\right\}=\psi^{-1}\left(Z\left(B_{1}\right)\right)$. By direct calculation $\left[\bar{s}_{m-3}, \tau\right] \in \bar{G}_{m-2}=Z\left(B_{0}\right)$. Hence as $\bar{s}_{m-3} \in Z(B), Z_{2}\left(B_{0}\right)=$ $\bar{G}_{m-3} A_{m-1}=\psi^{-1}\left(Z\left(B_{1}\right)\right)$ and $Z_{2}\left(B_{0}\right)=\psi^{-1}\left(Z\left(B_{1}\right)\right)$. Thus $B_{0} / Z_{2}\left(B_{0}\right) \cong B_{1} / Z\left(B_{1}\right)$ and $Z_{i}\left(B_{0} / Z_{2}\left(B_{0}\right)\right) \cong Z_{i}\left(B_{1} / Z\left(B_{1}\right)\right)$. Consequently $Z_{i}\left(B_{0}\right)=\bar{G}_{m-i+1} A_{m-i-1}$.

4. $p$-groups of maximal class. By definition a $p$-group of maximal class is a $p$-group of type $(m, 1)$. In this case $G_{i} / G_{i+1}$ is of order $p$ for $1 \leqslant i \leqslant m-1$ and also $A_{i} / A_{i+1}$ is of order $p$. This makes it possible to strengthen the results of the previous sections.

(4.1) Proposition. Let $G$ be a p-group of type $(m, n), m \geqslant 4$.

(a) $G$ can be embedded in a p-group $H$ of type $(m+1, n)$ if and only if $G$ has an automorphism $\tau$ such that

(1) $\tau: s \rightarrow s s_{1}^{\alpha}, \tau: s_{1} \rightarrow s_{1} u$, where $\alpha \in \mathbf{Z}, 1 \leqslant \alpha \leqslant p-1,(\alpha, p)=1$ and $u \in G_{3}$.

(2) $\tau^{p^{n}} \in \bar{G}, \tau^{p^{n-1}} \notin \bar{G}$. 
(b) Assume that $G$ has degree of commutativity $k=1$. If $m \leqslant p+1$ and $\tau \in \operatorname{Aut}(G)$ satisfies (1) of part (a), then $\tau$ satisfies (2) as well.

Proof. (a) If $G$ is embedded in a $p$-group $H$ of type $(m+1, n)$ then $H$ is generated by two elements $s$ and $\sigma_{1}$ with $\left[s, \sigma_{1}\right]=s_{1}^{-1}$. So the automorphism induced on $G$ by $\sigma_{1}$ satisfies (1) and (2) of part (a) of the proposition. Assume that $G$ has an automorphism $\tau$ which satisfies (1) and (2). Then by (2) and the definition of $\tau, H / G$ is cyclic of order $p^{n}$. We prove by induction on $|H|$ that $H_{m-i}=G_{m-i-1}$, for $i \geqslant 0$. $H_{m}$ is generated by $\left\{\left[\tau, s, x_{1}, \ldots, x_{m-2}\right]\right\}$ where $x_{t} \in\{\tau, s\}$. Since $[\tau, s] \equiv s_{1}^{\alpha} \bmod G_{2}$ and $\left[s_{1}, \tau\right] \in G_{3}$, it follows that if one of the $x_{i}$ 's is $\tau$ then $\left[\tau, s, x_{1}, \ldots, x_{m-2}\right] \in G_{m}$ $=1$. Hence $H_{m}=\langle[\tau,(m-1) s]\rangle=G_{m-1}$. Hence by the induction hypothesis for $G / G_{m-1}$ we get $H_{m-1} / H_{m}=K_{m-i-1}\left(G / G_{m-1}\right)=G_{m-i-1} / G_{m-1}=G_{m-i-1} / H_{m}$ for every $i \geqslant 1$. Consequently $H_{m-i}=G_{m-i-1}$ for $i \geqslant 1$ and $H$ is of type $(m+1, n)$, by definition.

(b) Since $s^{\tau^{p^{n-1}}}=s\left[s, \tau^{p^{n-1}}\right]=s[s, \tau]^{p^{n-1}} \bmod G_{2}$ by the collection formula, $s^{\tau^{p^{n-1}}}$ $\equiv s s_{1}^{\alpha^{p^{n-1}}} \bmod G_{2}$ for every $\tau$ which satisfies (1) of part (a). Since $[s, g] \in G_{2}$ by (1.3) this implies that $[s, \bar{g}] \in G_{2}$; hence $\tau^{p^{n-1}} \notin \bar{G}$. Thus we prove $\tau^{p^{n}} \in \bar{G}$.

By the collection formula $s_{1}^{\tau^{p^{n}}}=s_{1}\left[s_{1}, \tau^{p^{n}}\right]=s_{1}\left[s_{1} \tau\right]^{p^{n}} c_{2}^{\left(p^{n}\right)} \ldots c_{p^{n}}$, where $c_{i} \in$ $K_{i}\left(\left\langle\left[s_{1}, \tau\right], \tau\right\rangle\right)$ for $i \geqslant 2$. Since $u=\left[s_{1}, \tau\right] \in G_{3},\left[s_{1}, \tau, \tau\right] \leqslant\left[G_{3}, \tau\right]$. Now, $s_{2}^{\tau}=$ $\left[s_{1}, s\right]^{\tau}=\left[s_{1} u, s s_{1}^{\alpha}\right]=s_{2} v$ where $v \in G_{4}$ and by induction on $i$ we see that $\left[s_{i}, \tau\right] \in G_{i+2}$. Hence $K_{i}\left(\left\langle\left[s_{1}, \tau\right], \tau\right\rangle\right) \leqslant G_{i+2}$. In particular, $c_{p} \in G_{p+2}=1$ and $s_{1}^{\tau^{p^{n}}}$ $=s_{1} u^{p^{n}}=s_{1}$, as $\exp \left(G_{3}\right)=p^{n}$ by (1.5). By a similar application of the collection formula we get $s^{\tau^{p^{n}}}=s\left(s_{1}^{\alpha}\right)^{p^{n}}=s s_{p}^{\beta}$, by (1.5). We claim that $\tau^{p^{n}}=\bar{s}_{p-1}^{-\beta}$. Indeed, $\left[s_{1}, \bar{s}_{p-1}^{-\beta}\right] \in G_{p+1}=1$ as $G$ has degree of commutativity $\geqslant 1$ and $\left[s, \bar{s}_{p-\frac{1}{p^{n}}}^{-\beta}\right]=$ $\left[s, \bar{s}_{p-1}\right]^{-\beta}=\left[s_{p-1}, s\right]^{\beta}=s_{p}^{\beta}$. Hence with $\bar{g}=\bar{s}_{p-1}^{-\beta}$ we get $s^{\bar{g}}=s^{\tau^{p^{n}}}, s_{1}^{\bar{g}}=s_{1}^{\tau^{p^{n}}}$ and $\tau^{p^{n}} \in \bar{G}$, as required.

(4.2) Theorem. Let $G$ be a p-group of maximal class of order $p^{m}, P$ the Sylow p-subgroup of $\operatorname{Aut}(G)$ and $B=\left\{\sigma \in P \mid[s, \sigma],\left[s_{1}, \sigma\right] \in G_{2}\right\}$.

(a) If $G$ can be embedded in a p-group of maximal class $G_{0}$ of class $m$ then $P=\bar{G}_{0} B$, $|P / B|=p$.

(b) If $G / G_{p+1}$ cannot be embedded in a p-group of maximal class of order $p^{p+1}$ and $G$ has degree of commutativity $\geqslant 1$ then $P=B$.

(c) If $m \geqslant 3 p+6$ then $\left|A_{3}\right| \geqslant p^{[(m-3 p+8) / 2]}$ for $p>3$ and $\left|A_{3}\right| \geqslant 3^{[(m+1) / 2]}$ for $p=3$. Here $A_{3}=\left\{\sigma \in B \mid[s, \sigma]=1,\left[s_{1}, \sigma\right] \in G_{3}\right\}$ and $[a]$ is the integral part of $a$, for every $a \in \mathbf{Q}$.

Proof. (a) By (1.1) $P / B$ is isomorphic to a subgroup of

$$
\left\{\left(\begin{array}{c}
1, c \\
0,1
\end{array}\right) \mid c \in \mathbf{Z}_{p}\right\}
$$

If $G$ can be embedded in $G_{0}$ then $B \neq P$ by Proposition 4.1 ; hence $P=\bar{G}_{0} B$ and $|P / B|=p$.

(b) If $G / G_{p}$ cannot be embedded in a $p$-group of maximal class of order $p^{p+1}$ then $G$ has no automorphism $\tau$ such that $[s, \tau] \in G_{1} / G_{2}$ and $\left[s_{1}, \tau\right] \in G_{3}$, by Proposition 4.1. As every $\tau \in P / B$ would move $s$ to $s s_{1}^{\alpha} \bmod G_{2}$, this means that $P=B$. 
(c) Assume that $G$ has degree of commutativity $k$. If $i$ is the smallest $j$ such that $\left[s_{2}, s_{j}\right]=1$ then $i+k+1=m$, i.e. $i=m-k-1$. For $m \geqslant 3 p-6,2 k \geqslant m-3 p$ +6 by [3] or [9]. Hence for $m \geqslant 3 p-6, i \leqslant m-1-(m-3 p+6) / 2 \leqslant$ $[(m-8+3 p) / 2]$. Hence if $i_{0}=[(m-8+3 p) / 2]$ then $G_{i_{0}} \leqslant Z\left(G_{1}\right)$ and the result follows by Proposition 2.1.

(4.3) THEOREM. Let $G$ be a metabelian p-group of maximal class of order $p^{m}, m \geqslant 4$. Let $P$ be the Sylow p-subgroup of $\operatorname{Aut}(G)$ and for $i \geqslant 3$ let $A_{i}=\{\sigma \in P \mid[s, \sigma]=1$, $\left.\left[s_{1}, \sigma\right] \in G_{i}\right\}$. Then

(a) $A_{i} \cong G_{i}$ for $i \geqslant 3$.

(b) $P$ is generated by $p+1$ elements.

(c) If $G$ can be embedded in a p-group of maximal class of order $p^{m+1}$ then $K_{i}(P)=\bar{G}_{i-1} A_{(i-1)(k-1)+3}$ and $Z_{i}(P)=A_{m-i+1} \bar{G}_{m-i-1}$, for $2 \leqslant i \leqslant m-2$.

(d) If $G / G_{p+1}$ cannot be embedded in a p-group of maximal class then $K_{i}(P)=\bar{G}_{i}$ and $Z_{i}(P)=A_{m-i} \bar{G}_{m-i-1}$.

Proof. (a) Let $R, J=J(R), \phi$ and $\theta$ be as in Lemma 1.11, let $x=\phi(\bar{s})-1$ and $H=x^{2} R$. Then for every $u \in H, u^{p} \in p H$; for $(x+1)^{p}=1$ implies that $x^{p}=p x r$, $r \in R$. Therefore if $u=f(x), f(t)=\sum_{i=2}^{w} a_{i} t^{i}, f(t) \in t^{2} Z[t]$, then $u^{p} \equiv$ $\sum_{i=2}^{w} a_{i}^{p} x^{i p} \bmod p x^{2} R$; hence $u^{p} \equiv 0 \bmod p x^{2} R$, i.e. $u^{p} \in p H$. Thus $(1+u)^{p} \in 1+$ $p H$ and $\mho_{1}(1+H) \leqslant 1+p H$. Since $\theta$ sends $H$ on $G_{4}, H$ is generated as an abelian group, by $x^{2}, x^{3}, \ldots, x^{p}$ by (1.5) and (1.6) and it follows by induction on $|G|$ that $1+x^{2}, \ldots, 1+x^{p}$ generate $1+H$. Hence $H \cong 1+H$ by Lemma 1.11(f). This means that $A_{3} / A_{m-1} \cong H \cong G_{4}$. Since $G_{4} \cong G_{3} / G_{m-1}$ by 1.9 (b) and (1.10), $G_{3} / G_{m-1} \cong A_{3} / A_{m-1}$. We claim that if $\sigma \in A_{i} / A_{i+1}$ then $|\sigma|=\left|s_{i}\right|, m-1 \leqslant i \leqslant 3$. Indeed, by the collection formula $\left[s_{1}, \sigma^{p}\right]=\left[s_{1}, \sigma\right]^{p} c_{2}^{(p)} \ldots c_{p}$ where $c_{j} \in$ $K_{j}\left(\left\langle\left[s_{1}, \sigma\right], \sigma\right\rangle\right) \leqslant G_{i j}$. Hence $\left[s_{1}, \sigma^{p}\right] \equiv\left[s_{1}, \sigma\right]^{p} \bmod G_{i p} \mho\left(G_{2 i}\right)$. Since $\mho_{1}\left(G_{2 i}\right)$ $=G_{2 i+p-1}$ by $(1.5)$ and $2 i+p-1, p i \geqslant i+p$ for $i \geqslant 2$, we have $\left[s_{1}, \sigma^{p}\right] \equiv$ $\left[s_{1}, \sigma\right]^{p} \bmod G_{i+p}$, i.e. $\left[s_{1}, \sigma^{p}\right] \equiv u^{p} \bmod G_{i+p}$, where $u=\left[s_{1}, \sigma\right] \in G_{i} / G_{i+1}$. But as $u^{p} \in G_{i+p-1} / G_{i+p}$ by (1.5), this means that $\left[s_{1}, \sigma^{p}\right] \in G_{i+p-1} / G_{i+p}$ and our claim follows. In particular, $G_{3}$ and $A_{3}$ have the same exponent $p^{e}$, say, and to every $1 \leqslant i \leqslant e, \mho_{i}\left(A_{3}\right)=A_{m-i(p-1)}$. If $e=1$ then $A_{3}$ and $G_{3}$ are elementary abelian of the same order, hence isomorphic. If $e \geqslant 2$, then $G_{m-1} \mho_{i}\left(G_{3}\right)$ and by our claim $A_{m-1} \leqslant \mho_{i}\left(A_{3}\right)$ for $1 \leqslant i \leqslant e-2$. Thus, $A_{3} / \mho_{i}\left(A_{3}\right) \cong G_{3} / \mho_{i}\left(G_{3}\right)$ for $1 \leqslant i \leqslant e-1$. But then $\mho_{i}\left(A_{3}\right) \cong \mho_{i}\left(G_{3}\right)$ for $1 \leqslant i \leqslant e-1$ and as $\exp \left(A_{3}\right)=\exp \left(G_{3}\right)=p^{e}$ and $\left|A_{3}\right|=\left|G_{3}\right|$ we obtain $A_{3} \cong G_{3}$. By (1.10) this implies $A_{i} \cong G_{i}$ for $i \geqslant 3$.

(b) $A_{3}$ is generated by $p-1$ elements. By Theorem 4.2 either $P=\bar{G} A_{3}$ or $P=\bar{G} A_{3}\langle\tau\rangle$, where $[\tau, \bar{s}] \equiv \bar{s}_{1} \bmod \bar{G}_{2} A_{3}$. Hence in any case $P$ can be generated by $p-1+2=p+1$ elements.

(c) By Theorem 3.2(f) and (d) $Z_{i}(P)=A_{m-i+1} \bar{G}_{m-i-1}$ and $\bar{G}_{i-1} \leqslant K_{i}(P) \leqslant$ $A_{(i-1)(k-1)+3} \bar{G}_{i-1}$. Since $\left|G_{i} / G_{i+1}\right|=p$ for $2 \leqslant i \leqslant m-1$, it follows from Lemma 3.1(d) that $\left[\tau, A_{i}\right] \equiv A_{i+k-1} \bmod \bar{G}_{i-1}$; hence $K_{i}(P) \equiv A_{(i-1)(k-1)+3} \bmod \bar{G}_{i-1}$, and the result follows.

(d) By Theorem 4.2(b) $P=A_{3} \bar{G}$. Hence the result follows from Theorem 3.2(e). 


\section{REFERENCES}

1. N. Blackburn, On a special class of p-groups, Acta Math. 100 (1958), 45-92.

2. J. A. Gallian, Finite p-groups with homocyclic central factors, Canad. J. Math. 26 (1974), 636-643.

3. C. R. Leedham-Green and Susan McKay, On p-groups of maximal class. I, Quart. J. Math. Oxford Ser. (2) 27 (1976), 297-311.

4. B. Huppert, Endlich Gruppen. I, Springer-Verlag, Berlin, 1967.

5. A. Juhász, On finite groups with a Sylow p-subgroup of type ( $m, n)$, Israel J. Math. 36 (1980), 133-168.

6. On metabelian p-groups (unpublished).

7. H. Liebeck, The automorphism group of finite p-groups, J. Algebra 4 (1966), 426-432.

8. M. Lazard, Quelques calculs concernant la formule de Hausdorff, Bull. Soc. Math. France 91 (1963), 435-451.

9. R. Shepherd, p-groups of maximal class, Ph.D. Dissertation, Univ. of Chicago, 1970.

Mathematics INSTITUTE, UNiversity OF WaRWICK, COVENTRY CV4 7AL, England

Current address: Department of Mathematics, Hebrew University of Jerusalem, Jerusalem, Israel 